\title{
Temporal Modulation of a Spatially Periodic Potential for Kinetically Governed Oriented Motion
}

\author{
H. Berthoumieux, ${ }^{\dagger}$ L. Jullien, ${ }^{\dagger}$ A. Lemarchand ${ }^{\ddagger *}$ \\ $\dagger$ Ecole Normale Supérieure, \\ Département de Chimie, \\ UMR CNRS-ENS-Université Pierre et Marie Curie - Paris6 8640 PASTEUR, \\ 24, rue Lhomond, 75231 Paris Cedex 05, France. \\ E-mail: Ludovic.Jullien@ens.fr \\ $\ddagger$ Université Pierre et Marie Curie - Paris6, UMR 7600 LPTMC, Paris, F-75005 France; \\ CNRS, UMR 7600 LPTMC, Paris, F-75005 France. \\ E-mail: anle@lptmc.jussieu.fr
}

Corresponding author: A. Lemarchand, Université Pierre et Marie Curie - Paris 6, CNRS, UMR 7600, Laboratoire de Physique Théorique de la Matière Condensée, 4 place Jussieu, case courrier 121, 75252 Paris cedex 05, France.

Fax: 33(0)144277287, electronic mail: anle@lptmc.jussieu.fr

\section{Supporting Information}

- Results and discussion in the absence of a ramp during $T_{2}^{R}\left(E_{2}=0\right)$

- Supplementary Tables

- Table S-1: Sensitivity of the normalized velocity to variation of times $T_{1}$ and $T_{2}$ for a given set of parameters $\left(\Delta \psi=0.6, x_{1}=32, x_{2}=16, B_{0}=\right.$ $\left.1, D=0.01, T_{1}^{R}=11000, T_{2}^{R}=9000\right)$ with $E_{2}=0 . k_{1}^{\max }\left(T_{1}, T_{2}\right), k_{2}^{\max }\left(T_{1}, T_{2}\right)$, $K^{\max }\left(T_{1}, T_{2}\right), v^{\max }\left(T_{1}, T_{2}\right)$ (respectively $k_{1}^{R}\left(T_{1}^{R}, T_{2}^{R}\right), k_{2}^{R}\left(T_{1}^{R}, T_{2}^{R}\right), K^{R}\left(T_{1}^{R}, T_{2}^{R}\right)$, $\left.v^{R}\left(T_{1}^{R}, T_{2}^{R}\right)\right)$ designate the rate constants, the thermodynamic constant and the velocity associated to the species that exhibits the maximal velocity for $\left(T_{1}, T_{2}\right)$ (respectively $\left(T_{1}^{R}, T_{2}^{R}\right)$ ).

- Table S-2: Dependence of the normalized velocity $\frac{v}{v^{R}}$ on the rate constants $k_{1}$ and $k_{2}$ for the same parameters as in Table S-1 in the case of an alternating asymmetric sawtooth to flat potential at the period $T_{1}^{R}+T_{2}^{R}$ or of a permanent asymmetric sawtooth potential. 
- Table S-3: Dependence of the normalized apparent diffusion coefficient $\frac{D_{a p p}}{D}$ on the rate constants $k_{1}$ and $k_{2}$ for the same parameters as in Table S- 1 in the case of an alternating sawtooth to zero potential at the period $T_{1}^{R}+T_{2}^{R}\left(E_{2}=0\right)$.

- Supplementary Figures

- Figure S-1: Velocity $v$ as a function of $\log _{10} T_{1}$ for the resonant couple $\left(k_{1}^{R}, k_{2}^{R}\right)$ and for the negatively charged nonreactive species $\mathbf{A}_{n r}$ in the absence of a potential ramp $\left(E_{2}=0\right)$ during $T_{2}^{R}$.

- Figure S-2: Normalized velocity $v / v^{R}$ as a function of $\log _{10} k_{2}$ for $k_{1}=k_{1}^{R}$ (a) or of $\log _{10}\left(k_{1} / k_{1}^{R}\right)$ for $k_{2}=k_{2}^{R}$ (b)in the absence or in the presence of a potential ramp chosen to nullify the $\mathbf{A}_{n r}$ velocity during $T_{2}$. 
Results and discussion in the absence of a ramp during $T_{2}^{R}\left(E_{2}=0\right)$

We examine the characteristics of the resonance phenomenon in the absence of a field $E_{2}$ during $T_{2}^{R}$.

\section{Approximate analytical derivation}

In view of the regime of weak $E_{2}$ field for the considered sawtooth potential asymmetry, the approximate analytical expressions (13-16) and (18) with $E_{2}=0$ are relevant for $\Delta \psi$, $x_{1}, x_{2}, T_{1}^{R}, T_{2}^{R}$, and $B_{\text {eff }}$ in the absence of a ramp during $T_{2}^{R}$.

\section{Numerical results}

We numerically integrate the evolution equations (7-9) to directly obtain the velocity $v$ and the apparent diffusion coefficient $D_{a p p}$ of a mixture component $\{\mathbf{A}, \mathbf{C}\}$ submitted to the periodic potential sequence: $\psi^{1}(x)$ during $T_{1}, \psi^{2}(x)=0$ during $T_{2}$.

\section{Evaluation of the approximate analytical approach}

We evaluate the relevance of the approximate analytical approach for a given set of parameters $\left(\Delta \psi, x_{1}, x_{2}, B_{0}, E_{2}=0\right)$.

We first study the $v$ behavior for optimized values of times $T_{1}=T_{1}^{R}$ and $T_{2}=T_{2}^{R}$, respectively defined by Eqs. $(15,16)$. In the case $E_{2}=0$, the maximum velocity $v^{R}$ differs with only 1 percent with the value found in the case of a permanent potential. This result supports the relevance of the analytical definition of $T_{1}^{R}$ and $T_{2}^{R}$ as the times of migration oven the length $x_{1}$ and diffusion along the length $x_{2}$ respectively, when taking the reaction (1) into account.

Then we investigate the sensitivity of the resonance phenomenon on the values of the parameters $T_{1}$ and $T_{2}$, i. e. their influence on the position and the height of the velocity peak. For each $\left(T_{1}, T_{2}\right)$ couple considered in Table S-1, we look for the rate constant values, denoted $k_{1}^{\max }\left(T_{1}, T_{2}\right)$ and $k_{2}^{\max }\left(T_{1}, T_{2}\right)$, that lead to the maximal velocity $v^{\max }\left(T_{1}, T_{2}\right)$. The last column of Table S-1 compares $v^{\max }\left(T_{1}, T_{2}\right)$ with the resonant velocity $v^{R}\left(T_{1}^{R}, T_{2}^{R}\right)$ obtained for the values $\left(T_{1}^{R}, T_{2}^{R}\right)$ predicted in Eqs.(15,16). Table S-1 shows that $k_{1}^{\max }\left(T_{1}, T_{2}\right)$ and $k_{2}^{\max }\left(T_{1}, T_{2}\right)$ respectively depart from $k_{1}^{R}\left(T_{1}^{R}, T_{2}^{R}\right)$ and $k_{2}^{R}\left(T_{1}^{R}, T_{2}^{R}\right)$ upon varying $T_{2}$ at fixed $T_{1}=T_{1}^{R}$ or $T_{1}$ at fixed $T_{2}=T_{2}^{R}$.

The case $T_{1} / T_{2}>T_{1}^{R} / T_{2}^{R}$ favors the couples $\left\{\mathbf{A}_{\ell}, \mathbf{C}_{\ell}\right\}$ with large equilibrium constants $K_{\ell}$ that are in average mainly in their neutral state $\mathbf{C}_{\ell}$. If $T_{1}>T_{1}^{R}$ and $T_{2}=T_{2}^{R}$, those species have enough time to reach the maxima of the $\psi^{1}(x)$ potential and the largest probability to cross the potential barrier during $T_{1} ; v^{\max }\left(T_{1}, T_{2}\right)$ is here lower 
than $v^{R}\left(T_{1}^{R}, T_{2}^{R}\right)$ because an essentially similar distance is covered in a longer time than $T_{1}^{R}+T_{2}^{R}$. If $T_{1}=T_{1}^{R}$ and $T_{2}<T_{2}^{R}$, there is not anymore enough time to cover by diffusion the distance $x_{2}$ during $T_{2}$ and the potential barrier has to be overcome during the $T_{1}$ phase; $v^{\max }\left(T_{1}, T_{2}\right)$ is again lower than $v^{R}\left(T_{1}^{R}, T_{2}^{R}\right)$.

In the opposite, $T_{1} / T_{2}<T_{1}^{R} / T_{2}^{R}$ favors the couples $\left\{\mathbf{A}_{\ell}, \mathbf{C}_{\ell}\right\}$ with small equilibrium constants $K_{\ell}$ that are in average mainly in the charged state $\mathbf{A}_{\ell}$. If $T_{1}=T_{1}^{R}$ and $T_{2}>T_{2}^{R}$, crossing the barrier in the neutral state during $T_{1}$ is not anymore an advantage because this can be easily done during $T_{2}$; diffusion dominates the motion and $v^{\max }\left(T_{1}, T_{2}\right)<$ $v^{R}\left(T_{1}^{R}, T_{2}^{R}\right)$. If $T_{1}<T_{1}^{R}$ and $T_{2}=T_{2}^{R}$, reaching the maxima of the $\psi^{1}(x)$ potential now requires to spend in average less time in the neutral state than for $\left\{\mathbf{A}^{R}, \mathbf{C}^{R}\right\}$; reducing $T_{1}$ here increases the maximal velocity because an essentially similar distance is covered in a smaller time than $T_{1}^{R}+T_{2}^{R}$. Nevertheless this trend is limited by the duration of migration over $x_{1}$ for a charged nonreactive species $T_{1}^{l i m}=\frac{x_{1}^{2}}{|\mu| \Delta \psi}$ : The motion of all the species vanishes for $T_{1}<T_{1}^{\text {lim }}$ (see Figure S-1 for an illustration).

Beyond validating the approximate analytical approach, the present numerical calculations show that obeying Eqs. $(15,16)$ for $T_{1}$ and $T_{2}$ is crucial to achieve the separation of the desired couple: A departure by a factor of ten is already significant.

\section{Selectivity of the separation process}

We now examine the selectivity of the separation method for optimized values $T_{1}^{R}$ and $T_{2}^{R}$. Figure S-2a and S-2b display the dependence of the velocity $v$ on the rate constants $k_{1}$ for $k_{2}=k_{2}^{R}$ and on $k_{2}$ for $k_{1}=k_{1}^{R}$ respectively. Table S-2 extracts some particular results.

The resonance phenomenon is present. In contrast to the situation encountered with a permanent sawtooth potential, the observed resonance is not symmetric in the $\left\{k_{1 \ell}, k_{2 \ell}\right\}$ space. One may notice that the species that are at the most interfering are associated with $K_{\ell} \leq K^{R}$ (see also Figure S-2a and S-2b). In fact, even the negatively charged nonreactive species $\mathbf{A}_{n r}$ moves only 15\% more slowly than the resonant couple. It is this detrimental behavior that led us to introduce a constant field during $T_{2}^{R}$ to slow down the couples mainly in the charged state (small values of $k_{1}$ and large values of $k_{2}$ ) and improve the selectivity of the sorting protocol. The location of the resonant species depends on the application of the field $E_{2}$ during $T_{2}^{R}$ : The value of $k_{2}^{R}$ derives from the geometry of the potential and is essentially independent on $E_{2}$ whereas the value of $k_{1}^{R}$ is affected by the variation of $B_{e f f}$. For the parameter values chosen in Figure S-2 the value of $k_{1}^{R}$ with or without the ramp varies by a factor of 10 .

Table S-3 eventually shows that the resonance phenomenon observed for velocity is not accompanied by a resonance for diffusion: the apparent diffusion coefficient is at most 
equal to the actual diffusion coefficient $D$ and often decreased. 
Table S-1. Sensitivity of the normalized velocity to variation of times $T_{1}$ and $T_{2}$ for a given set of parameters $\left(\Delta \psi=0.6, x_{1}=32, x_{2}=16, B_{0}=1, D=0.01, T_{1}^{R}=11000, T_{2}^{R}=\right.$ $9000)$ with $E_{2}=0 . k_{1}^{\max }\left(T_{1}, T_{2}\right), k_{2}^{\max }\left(T_{1}, T_{2}\right), K^{\max }\left(T_{1}, T_{2}\right), v^{\max }\left(T_{1}, T_{2}\right)$ (respectively $\left.k_{1}^{R}\left(T_{1}^{R}, T_{2}^{R}\right), k_{2}^{R}\left(T_{1}^{R}, T_{2}^{R}\right), K^{R}\left(T_{1}^{R}, T_{2}^{R}\right), v^{R}\left(T_{1}^{R}, T_{2}^{R}\right)\right)$ designate the rate constants, the thermodynamic constant and the velocity associated to the species that exhibits the maximal velocity for $\left(T_{1}, T_{2}\right)$ (respectively $\left.\left(T_{1}^{R}, T_{2}^{R}\right)\right)$.

\begin{tabular}{|c|c|c|c|c|c|}
\hline$\frac{T_{1}}{T_{1}^{R}}$ & $\frac{T_{2}}{T_{2}^{R}}$ & $\frac{k_{1}^{\max }\left(T_{1}, T_{2}\right)}{k_{1}^{R}\left(T_{1}^{R}, T_{2}^{R}\right)}$ & $\frac{k_{2}^{\max }\left(T_{1}, T_{2}\right)}{k_{1}^{R}\left(T_{1}^{R}, T_{2}^{R}\right)}$ & $\frac{K^{\max }\left(T_{1}, T_{2}\right)}{K^{R}\left(T_{1}^{R}, T_{2}^{R}\right)}$ & $\frac{v^{\max }\left(T_{1}, T_{2}\right)}{v^{R}\left(T_{1}^{R}, T_{2}^{R}\right)}$ \\
\hline 1 & 1 & 1.00 & 1.00 & 1.00 & 1.00 \\
\hline 1 & 10 & 0.013 & 20.3 & 0.0006 & 0.28 \\
\hline 10 & 1 & 5.6 & 0.16 & 35 & 0.24 \\
\hline 1 & 0.1 & 5 & 0.16 & 31.25 & 0.45 \\
\hline 0.1 & 1 & $0^{a}$ & $0^{a}$ & $0^{a}$ & 1.65 \\
\hline
\end{tabular}

${ }^{a}$ The fastest species is the negatively charged nonreactive species $\mathbf{A}_{n r}$. 
Table S-2. Dependence of the normalized velocity $\frac{v}{v^{R}}$ on the rate constants $k_{1}$ and $k_{2}$ for the same parameters as in Table S-1 in the case of an alternating asymmetric sawtooth to flat potential at the period $T_{1}^{R}+T_{2}^{R}$ or of a permanent asymmetric sawtooth potential.

\begin{tabular}{|c|c|c|c|}
\hline$k_{1}, k_{2}$ & $K / K^{R}$ & $E_{2}=0$ & Stationary potential \\
\hline$k_{1}^{R}, k_{2}^{R}$ & 1 & 1.00 & 1.00 \\
\hline$k_{1}^{R}, 10 k_{2}^{R}$ & 0.1 & 0.89 & 0.05 \\
\hline $10 k_{1}^{R}, k_{2}^{R}$ & 10 & 0.55 & 0.31 \\
\hline$k_{1}^{R}, 0.1 k_{2}^{R}$ & 10 & 0.36 & 0.03 \\
\hline $0.1 k_{1}^{R}, k_{2}^{R}$ & 0.1 & 0.95 & 0.51 \\
\hline $10 k_{1}^{R}, 10 k_{2}^{R}$ & 1 & 0.75 & 0.19 \\
\hline $0.1 k_{1}^{R}, 0.1 k_{2}^{R}$ & 1 & 0.47 & 0.30 \\
\hline $0.1 k_{1}^{R}, 10 k_{2}^{R}$ & 0.01 & 0.87 & 0.01 \\
\hline $10 k_{1}^{R}, 0.1 k_{2}^{R}$ & 100 & 0.25 & 0.03 \\
\hline $\mathbf{A}_{n r}$ & 0 & 0.85 & 0.00 \\
\hline
\end{tabular}


Table S-3. Dependence of the normalized apparent diffusion coefficient $\frac{D_{a p p}}{D}$ on the rate constants $k_{1}$ and $k_{2}$ for the same parameters as in Table S- 1 in the case of an alternating sawtooth to zero potential at the period $T_{1}^{R}+T_{2}^{R}\left(E_{2}=0\right)$.

\begin{tabular}{|c|c|c|}
\hline$k_{1}, k_{2}$ & $K / K^{R}$ & $\frac{D_{\text {app }}}{D}$ \\
\hline$k_{1}^{R}, k_{2}^{R}$ & 1 & 0.78 \\
\hline$k_{1}^{R}, 10 k_{2}^{R}$ & 0.1 & 0.57 \\
\hline $10 k_{1}^{R}, k_{2}^{R}$ & 10 & 0.77 \\
\hline$k_{1}^{R}, 0.1 k_{2}^{R}$ & 10 & 1.03 \\
\hline $0.1 k_{1}^{R}, k_{2}^{R}$ & 0.1 & 0.68 \\
\hline $10 k_{1}^{R}, 10 k_{2}^{R}$ & 1 & 0.55 \\
\hline $0.1 k_{1}^{R}, 0.1 k_{2}^{R}$ & 1 & 0.97 \\
\hline $0.1 k_{1}^{R}, 10 k_{2}^{R}$ & 0.01 & 0.55 \\
\hline $10 k_{1}^{R}, 0.1 k_{2}^{R}$ & 100 & 1.03 \\
\hline $0,0\left(\mathbf{A}_{n r}\right)$ & 0 & 0.55 \\
\hline
\end{tabular}



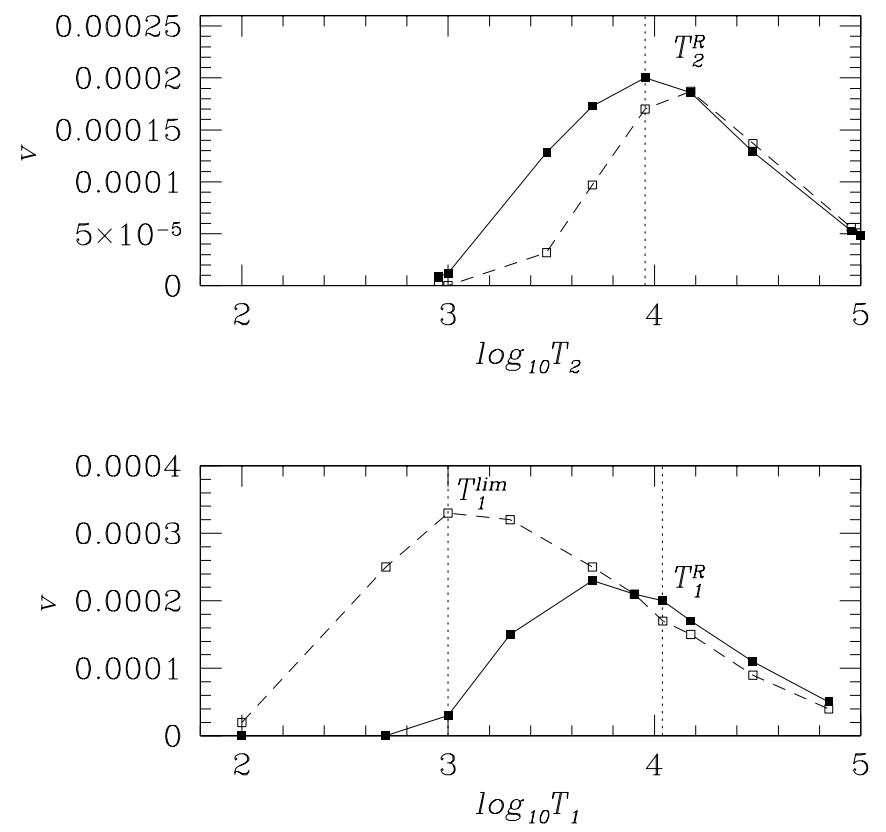

Figure S-1. Velocity $v$ as a function of $\log _{10} T_{1}$ for the resonant couple $\left(k_{1}^{R}, k_{2}^{R}\right.$ ) (solid squares and solid line) and for the negatively charged nonreactive species $\mathbf{A}_{n r}$ (open squares and dashed line $)$ in the absence of a potential ramp $\left(E_{2}=0\right) \operatorname{during} T_{2}^{R}(\Delta \psi=$ $\left.0.6, x_{1}=32, x_{2}=16, B_{0}=1, D=0.01, T_{2}^{R}=9000\right)$. The vertical dotted lines give the analytical predictions for $T_{1}^{l i m}$ and $T_{1}^{R}$. The $\mathbf{A}_{n r}$ velocity is maximal for $T_{1}=T_{1}^{\text {lim }}$ and the resonant $\left\{\mathbf{A}^{R}, \mathbf{C}^{R}\right\}$ couple is faster than $\mathbf{A}_{n r}$ for $T_{1} \geq T_{1}^{R}$. We observe a good agreement between the analytical predictions of $T_{1}^{l i m}$ and $T_{1}^{R}$ and the corresponding values deduced from the numerical integration of Eqs.(7-9). 

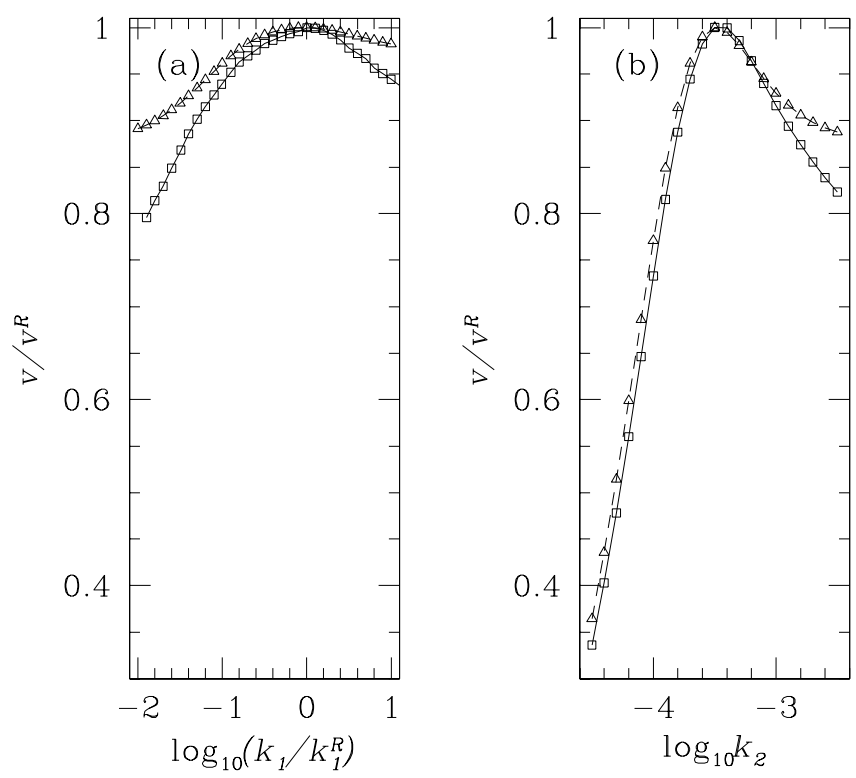

Figure S-2. Normalized velocity $v / v^{R}$ as a function of $\log _{10} k_{2}$ for $k_{1}=k_{1}^{R}$ (a) or of $\log _{10}\left(k_{1} / k_{1}^{R}\right)$ for $k_{2}=k_{2}^{R}(\mathbf{b})$. Triangles and dotted line: No potential $\operatorname{ramp}\left(E_{2}=0\right)$ during $T_{2}$; squares and solid line: potential ramp $E_{2}=0.000625$ chosen to nullify the $\mathbf{A}_{n r}$ velocity. Same parameters as in Fig. S-1 and $T_{1}=T_{1}^{R}=11000$. The results were obtained from numerical integration of Eqs (7-9). 